\title{
Art of Hysteroscopy
}

\author{
Nilgün Öztürk Turhan* \\ Department of Obstetrics and Gynecology, Fatih University Faculty of Medicine, Turkey
}

Submission: October 05, 2018 ; Published: October 25, 2018

*Corresponding author: Nilgün Öztürk Turhan, Department of Obstetrics and Gynecology, Fatih University Faculty of Medicine, İstanbul-Turkey, Turkey, Email: nilgunturhan@hotmail.com

\section{Abstract}

Hysteroscopy is a minimally invasive approach to view and treat pathologies within the uterine cavity, tubal ostia and cervical canal in both premenopausal and postmenopausal patients. Polyps, leiomyomas, intrauterine synechia, hyperplasia, malignancy, foreign bodies, retained products of conception and congenital uterine anomalies can both be diagnosed and treated by hysteroscopy.

Keywords: Diagnostic hysteroscopy; Operative hysteroscopy; Complications of hysteroscopy

\section{Introduction}

Hysteroscopy is an endoscopic surgical procedure performed for evaluation or treatment of the endometrial cavity, tubal ostia, or endocervical canal problems. It has a broad spectrum of indications and must be learned by all gynecologists. Indications of hysteroscopy are: for diagnostic purposes such as premenopausal or postmenopausal uterine bleeding, evaluation of infertility, recurrent miscarriage and for treatment purposes such as for restoration of uterine cavity in intrauterine synechia cases, congenital uterine anomalies, removal of intrauterine polyps and fibroids and for retrieval of a lost intrauterine contraceptive device (IUD), hysteroscopic sterilization, retained products of conception, endometrial ablation. Cervical cancer, heavy uterine bleeding, pelvic inflamatory disease, active herpetic infection and pregnancy are absolute contraindications for hysteroscopy.

\section{Equipments for Hysteroscopy}

Diagnostic and operative hysteroscopes can vary from traditionally available rigid diagnostic $(3 \mathrm{~mm})$ and operative ( $5 \mathrm{~mm})$ hysteroscopes to more complex state-of-the-art modern equipment including flexible fibrescopes, miniaturised semirigid/rigid diagnostic hysteroscopes and rigid operative (3.8$5 \mathrm{~mm}$ ) hysteroscopes. Flexible hysteroscopes range in diameter from $2.7-5 \mathrm{~mm}$ and the flexiple tip deflects in two directions ranging from 100 degrees to 180 degrees. Rigid telescopes are available in different angles of vision ranging from 0 to 30 degrees. Most diagnostic scopes are $30^{\circ} \mathrm{C}$ allowing a thorough inspection of uterine walls, cornual recesses and tubal ostia with minimal movement of the shaft. Their external diameters vary from $1.2 \mathrm{~mm}$ to $4 \mathrm{~mm}$. The single flow sheath of the rigid hysteroscope is used in combination with a 4 - to $5 \mathrm{~mm}$ outer sheath to create a continuous flow system and permit the passage of semirigid instruments such as scissors, graspers and biopsy forceps for endometrial biopsy, tubal cannulation, or intrauterine surgery.

Operative hysteroscopes are used to remove endocervical or endometrial lesions or to perform an endometrial ablation/ resection. Operative hysteroscopes typically range from $8 \mathrm{~mm}$ to $10 \mathrm{~mm}$ in diameter and contain a retractable hand piece wherein electrosurgical tips, laser devices, or mechanical instruments may be attached. Currently, there are three types of operative hysteroscopes: Operative sheath with instruments inserted through channels or fixed to the sheath, electrosurgical resectoscope and hysteroscopic morcellator [1].

Different types of energy have been used for operative hysteroscopy including laser, unipolar or bipolar energies. Laser is rarely used in modern hysteroscopic procedures. The most commonly used energy for hysteroscopic surgery is unipolar energy. To avoid dispersion of the electrical current, a lowviscosity distending medium such as $1.5 \%$ glycine is needed. Compared to normal saline, its use is associated with a high risk of fluid overload. Accordingly, a versatile bipolar electrosurgery system was developed allowing operative hysteroscopy using normal saline as a distending medium. As the electrical current is confined, it is safer than a unipolar device. This bipolar device can be used for cutting, coagulation or tissue vaporization including treatment of large endometrial polyps and submucosal myomas under $2 \mathrm{~cm}$.

Various novel methods of producing global endometrial ablation and smaller hysteroscopic morcellator devices are appearing and undergoing evaluation for safety and effectiveness [2]. They all have short treatment times, with acceptable pain 
profiles when used without general anaesthesia and require little, if any, cervical dilatation, suggesting that they may be appropriate for use in outpatient settings. Hysteroscopic morcellation appears efficient at removing prominent submucosal fibroids, but as safety and effectiveness have yet to be firmly established, procedures should be carefully audited for complications and treatment outcome [2].

\section{Uterine Distention}

Hysteroscopy enables visualisation of the uterine cavity and allows the diagnosis and surgical treatment of intrauterine pathology. To achieve this, the uterine cavity needs to be distended by a medium which could either be fluid or carbon Dioxide.

\section{Carbon Dioxide Gas}

Carbon dioxide $\left(\mathrm{CO}_{2}\right)$, a colorless gas, is used in outpatient settings for diagnostic purposes only as bleeding during operative procedures obscures visibility. The advantages of its use include the ease of cleaning and maintaining equipment and a clear view of the cavity in the absence of active bleeding or bubbles. Most gynecologists have abandoned the use of $\mathrm{CO}_{2}$ gas. The mixture of $\mathrm{CO}_{2}$ gas and fluid such as blood produces bubbles that impair visualization. In addition, it carries the risk of air embolism.

\section{Fluid Media}

Fluid media are used for operative procedures, as they allow continuous irrigation giving a clear picture and enable use of both mechanical and electrosurgical instruments. Many distending media have been used for hysteroscopy. Generally, normal saline is preferred to carbon dioxide as a distension medium as it allows improved image quality, permits quicker procedure, reduces the vasovagal episodes and has an added advantage of acting as a conducting medium for the use of bipolar energy for operative hysteroscopy. Normal saline bags of 1- and 3-1 volumes should be stocked. It is better to use warmed saline (room temperature)

Delivery of the distension medium can be safely and effectively achieved using: simple gravity, pressure bags or automated delivery systems. It is practical to use pressure bags with a squeeze bulb to achieve adequate uterine distension (80$120 \mathrm{mmHg}$ ) with normal saline during routine hysteroscopy. Automated pressure delivery system is recommended in order to maintain a constant intrauterine pressure and a clear view intraoperatively as well as accurate fluid deficit surveillance which is advantageous for prolonged cases such as endometrial resection or hysteroscopic myomectomy.

Fluid distending media can be classified into two main types: electrolyte-rich (conducting) media, for example, normal saline; and electrolyte-poor (nonconducting) media. However, it is now possible to use electrolyte media with bipolar electrosurgical systems. It also is important to understand which media are hypo-osmolar.

\section{Electrolyte-poor fluid}

Electrolyte-Poor fluids include glycine, 1.5\% (osmolality 200 $\mathrm{mOsmol} / \mathrm{kg}$-hypo-osmolar); sorbitol, 3\% (hypo-osmolar); and mannitol, $5 \%$ (which is iso-osmolar to plasma; $285 \mathrm{mOsmol} / \mathrm{kg}$ ). Glycine $1.5 \%$ is one of the most commonly used nonconducting media. Devices that use monopolar energy such as the traditional resectoscope must only be used with nonconducting media to prevent potential injury to the patient. They are compatible with radio-frequency energy, which cuts, desiccates, and fulgurates intrauterine tissue. Excessive absorption of hypo-osmolar fluids can cause hyponatremia, hyperammonemia, and decreased serum osmolality, with the potential for seizures, cerebral edema, and death. Some clinicians have recommended mannitol, $5 \%$, which is iso-osmolar and acts as its own diuretic. It may cause hyponatremia, but not decreased serum osmolality.

The use of a hyperosmolar solution of $32 \%$ dextran 70 has been abandoned. Its use even in low volumes has been associated with vascular overload and subsequent heart failure, pulmonary oedema and anaphylaxis. Further, it tends to caramelize quickly on instruments leading to damage.

\section{Electrolyte-containing fluid}

Normal saline solution and lactated Ringer's solution are readily available and are isotonic. These solutions can be used during diagnostic hysteroscopy and in operative cases where mechanical, laser, or bipolar energy is used. The risk of hyponatremia and decreased serum osmolality is low but careful attention should be paid to fluid input and output, with particular attention to the fluid deficit as pulmonary edema and congestive heart failure can still occur.

\section{Light Source and light head}

Most light sources are either Tungsten or cold xenon (175 watt). Either type of lamp provides adequate illumination for operative procedures, photography, and videotaping for hysteroscopy. Xenon lamps are more expensive than halogen ones. Illumination is primarily a function of the power of the light and the light transmission properties of the light head, but it is also influenced by the size and tissue propertises of the light head. Light heads are either Fibre optic or Liquid. The light cables are rather fragile, and they should be handled with extreme care to avoid damage to the optical fibres.

\section{Preoperative Considerations}

Patient preparation should start from the patient's first presentation. Patient and physician should discuss the hysteroscopic procedure, its risks and benefits. In premenopausal women with regular menstrual cycles, the optimal timing for hysteroscopic procedures is during the early proliferative stage (menstrual days 4-10), when the endometrial lining is the thinnest. Some women with unpredictable menses can be scheduled at any time for operative hysteroscopy. Cervical softening with 400 to $800 \mu \mathrm{g}$ of synthetic E1 prostaglandin 
(i.e., misoprostol) administered vaginally 12 hours before the procedure is not routinely recommended.

A systematic review and meta-analysis of randomized controlled trials on the effectiveness of cervical ripening with misoprostol administration before hysteroscopy showed that misoprostol had a significant effect on cervical ripening before hysteroscopy, except in the postmenopausal population but adverse effects were significantly more common with misoprostol than with placebo (risk difference $0.07,95 \% \mathrm{CI}$ 0.01-0.12) [3]. It is associated with increased side effects (e.g., abdominal pain, nausea, diarrhea, fever, and vaginal bleeding), no significant difference in rates of cervical laceration and false tract formation and the risk of overdilation of the cervix for the small diameter office hysteroscopes.

\section{Office Hysteroscopy}

Office hysteroscopy is used for either diagnostic or minor operative procedures including abnormal uterine bleding, submucous fibroids, endometrial polyps and intrauterine synchecia, removal of uterine septum. Office hysteroscopy is safe, with a low incidence of serious complications and failure rate. Performing the procedure in the office with no speculum, tenaculum, or anesthesia will enable the patient comfort so that the patient can return to normal activities at the earliest. Major barriers to successful office hysteroscopy include pain, cervical stenosis, and poor visualization of the cervix. Therefore, preoperative patient selection and counseling are very important. Poor candidates for office hysteroscopy include patients who have cervical stenosis, high levels of anxiety, comorbidities, limited mobility, or significant uterine pathology requiring operative procedures. Minimal distention pressure for adequate visualization will reduce patient discomfort. Performing any procedure requiring $>3 \mathrm{~L}$ of fluid unless a weighted monitoring system is available is not recommended.

Equipment generally needed to perform hysteroscopic procedures in the office includes a hysteroscope with an outer sheath of less than $5 \mathrm{~mm}$ in outer diameter, distending media and infusion system, operative instrumentation, and a light source. Although it is possible for the surgeon to look directly into the eyepiece, cameras and video monitoring systems make it possible to obtain photographs and video and enable the patient to see images.

\section{Operative Hysteroscopy}

Operative hysteroscopy incorporates the use of mechanical, electrosurgical, or laser instruments to treat intracavitary pathologies such as removal of endometrial polyps, submucous fibroids, septa and adhesions or to perform targeted biopsy or an endometrial ablation/resection. Resectoscopes typically consist of a 7 to $9 \mathrm{~mm}$ sheath. The hysteroscopic morcellator consists of a rotary blade that cuts lesions; tissue is then aspirated through the morcellator. The smaller hysteroscopic morcellators are used for endometrial polypectomy in an outpatient setting whereas larger hysteroscopic morcellators can remove prominent submucosal fibroids.

\section{Prevention and Management of Complications}

Complications from hysteroscopy are rare, but some are potentially life threatening. Hysteroscopic procedures are associated with a low number of adverse events with an incidence, reported from Germany of $0.24 \%$ of 21,676 cases [4]. The most common perioperative complications associated with operative hysteroscopy were uterine perforation $(0.12 \%)$, resection of fibroids had the highest risk of uterine perforation $(0.15 \%)$, fluid-overload syndrome occurred in $0.06 \%$. The incidence of intra-operative hemorrhage in operative hysteroscopy ranged between 0.03 and $0.1 \%$. In the absence of uterine perforation, heavy intra-operative bleeding occurred in $0.03 \%$ of the patients, in $83.3 \%$ cases during endometrial ablation. The incidence of intra-operative hemorrhage during endometrial ablation was $0.1 \%$, Endomyometritis was documented in $0.01 \%$ of hysteroscopic patients.

\section{Hemorrhage}

Hemorrhage may occur during hysteroscopic resection of the endometrium, leiomyomas, uterine septa, or synechiae and from cervical lacerations or uterine perforation. It may originate from endometrium, myometrium or from damage to surroding vessels. Delayed hemorrhage may be seen among patients with endometritis weeks after surgery. Bleeding from a specific site within the uterine cavity, with no suspicion of uterine perforation, can be controlled with electrosurgical coagulation is most cases. Women with diffuse bleeding should be evaluated for coagulopathy. If coagulation testing is normal and diffuse bleeding continues, it can be treated by placing a Foley catheter in the uterine cavity and then distending the bulb with 15 to $30 \mathrm{~mL}$ of water. Alternative strategies, such as injection of vasopressin into the cervical stroma or uterine compression can be attempted. In extreme cases, laparoscopic suturing of a perforation, hysterectomy, or uterine artery embolization may be necessary.

\section{Fluid Overload}

Complications related to distending media vary according to the patient population and the media used. Systemic absorption of distension media can occur either by intravasation or extravasation. The most common mechanism of absorption is intravasation whereby fluid enters the systemic circulation when blood vessels and venous sinuses within the myometrium are opened. Extravasation of fluid refers to the entry of distension media into the peritoneal cavity where it is then reabsorbed. This may occur through passage of the fluid through either the fallopian tubes or an unrecognized perforation.

Fluid absorption is affected by size and number of the lesions removed (Large or deep resections for type 1/2 fibroids or transcervical resection of endometrium), the depth of myometrial resection, the number of myometrial sinuses opened, 
the intrauterine pressure, and the duration of procedure [5].

The best way to limit excess fluid intravasation is to monitor the fluid deficit closely and frequently throughout the procedure. Calculation of the absorbed media should take place in a closed and preferably automated system. If automated system is not available, the volume should be measured and deficit should be calculated every 5 to 10 minures.

The Practice Guidelines of the American Association for Gynecologic Laparoscopists (AAGL) recommend a maximum fluid deficit of $1000 \mathrm{~mL}$ of low-viscosity medium for healthy patients. The patient should be carefully evaluated and the procedure should be terminated expeditiously. For elderly patients and others with co-morbid conditions, a maximum fluid deficit of $750 \mathrm{~mL}$ is advised. The maximum fluid deficit for normal saline is unclear, but $2500 \mathrm{~mL}$ has been advocated [6].

Guidelines for fluid monitoring and the limits of fluid excess have been published and adapted by the American College of Obstetricians and Gynecologists' Committee on Gynecologic Practice as follows [1].

a) Intravenous hydration of patients undergoing hysteroscopy should be closely monitored preoperatively and intraoperatively. Hysteroscopic fluid absorption should be closely monitored intraoperatively.

b) Lower fluid deficit thresholds should be considered for elderly patients, patients with comorbid conditions, patients with cardiovascular or renal compromise, and when procedures take place in an outpatient setting with limited acute care and laboratory services.

c) In healthy patients, the maximum fluid deficit is $1,000 \mathrm{~mL}$ for hypotonic solutions, $2,500 \mathrm{~mL}$ for isotonic solutions, and $500 \mathrm{~mL}$ for high-viscosity solutions. However, if fluid $1,000 \mathrm{~mL}$ of a hypotonic solution, $2,000 \mathrm{~mL}$ of an electrolyte solution, or $300 \mathrm{~mL}$ of a high-viscosity solution, consideration should be given to stop further infusion and conclude the procedure. Electrolytes should be assessed, administration of diuretics considered, and further diagnostic and therapeutic intervention begun as indicated.

d) In an outpatient setting with limited acute care and laboratory services, consideration should be given to discontinuing procedures at a lower fluid deficit threshold than indicated earlier.

e) An automated fluid monitoring system facilitates early recognition of excessive deficit in real-time totals.

f) An individual should be designated to frequently measure intake and outflow and report the deficit to the operative team.

\section{Perforation}

Uterine perforation is the most common complication of hysteroscopy. In a retrospective study of 5474 hysteroscopies, uterine perforation occurred in 15 cases $(0.27 \%)$, however, although the perforation rate was $0.06 \%$ for cases of diagnostic hysteroscopy, it was $1 \%$ for those procedures categorized as "operative hysteroscopy" [7].

Before performing hysteroscopy, the clinician should perform a pelvic examination to determine uterine position. Ultrasound guidance may be useful in difficult cases. Midline uterine perforation rarely leads to significant morbidity unless a laser or electrosurgical device is used. Lateral uterine perforations can lead to the development of a retroperitoneal hematoma, and cervical perforations can result in significant immediate or delayed bleeding. Laparoscopy may be useful to determine the extent of damage, including the existence of bowel injury or bladder injury.

\section{Embolization}

Embolism (air or carbon dioxide) can occur with any hysteroscopic technique and can cause cardiovascular collapse or pulmonary edema. Clinically significant gas embolism appears to occur frequently in patients undergoing operative hysteroscopy. Preventive strategies include flushing air from tubing and making sure that the procedure is stopped and tubing is purged of air when bags are changed. To minimize the risk of gas embolization, the flow of $\mathrm{CO} 2$ should be limited to $100 \mathrm{~mL} /$ min with intrauterine pressure less than $100 \mathrm{~mm} \mathrm{Hg}$ and used with a hysteroscopic insufflator. Insufflators designed for use in laparoscopy must not be used for hysteroscopy.

\section{Infection}

Although hysteroscopy is performed through the "contaminated" environment the risk of infection is low. Studies report postoperative incidences of 0.01 to 1.42 percent incidence for endometritis [8].

\section{Conclusion}

Hysteroscopy is considered the gold standard for the evaluation and restoring of intracavitary problems in recent years with advanced experience of physicians, smaller diameter hysteroscopes, and common use of office-based procedures. It is minimally invasive and can be used with a high degree of safety. Knowledge of potential risks relating to distending media and intraoperative complications will enhance its safety.

\section{References}

1. (2018) ACOG Technology Assessment No. 13: Hysteroscopy. Obstet Gynecol 131(5): e151-e156.

2. Connor M (2015) New technologies and innovations in hysteroscopy. Best Pract Res Clin Obstet Gynaecol 29(7): 951-965.

3. Zhuo Z, Yu H, Jiang X (2016) A systematic review and meta-analysis of randomized controlled trials on the effectiveness of cervical ripening with misoprostol administration before hysteroscopy. Int J Gynaecol Obstet 132(3): 272-277.

4. Aydeniz B, Gruber IV, Schauf B, et al. (2002) A multicenter survey of complications associated with 21,676 operative hysteroscopies. Eur J Obstet Gynecol Reprod Biol 104(2): 160-164. 
5. McGurgan PM, Mcllwaine P (2015) Complications of hysteroscopy and how to avoid them. Best Pract Res Clin Obstet Gynaecol 29(7): 982-993.

6. Munro MG, Storz K, Abbott JA, Falcone T, Jacobs VR, et al. (2013) AAGL Practice Report: Practice Guidelines for the Management of Hysteroscopic Distending Media: (Replaces Hysteroscopic Fluid Monitoring Guidelines. J Am Assoc Gynecol Laparosc.2000;7:167-168. J Minim Invasive Gynecol 20(2): 137-148.

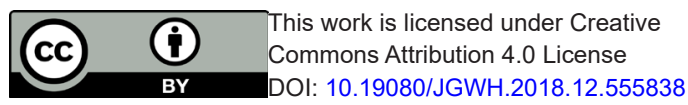

7. Kayatas S, Meseci E, Tosun OA, Arinkan SA, Uygur L, et al. (2014) Experience of hysteroscopy indications and complications in 5,474 cases. Clin Exp Obstet Gynecol 41(4): 451-454.

8. Kasius JC, Broekmans FJ, Fauser BC, Devroey P, Fatemi HM, et al. (2011) Antibiotic prophylaxis for hysteroscopy evaluation of the uterine cavity. Fertil Steril 95(2): 792-794.

\section{Your next submission with Juniper Publishers will reach you the below assets}

- Quality Editorial service

- Swift Peer Review

- Reprints availability

- E-prints Service

- Manuscript Podcast for convenient understanding

- Global attainment for your research

- Manuscript accessibility in different formats ( Pdf, E-pub, Full Text, Audio)

- Unceasing customer service

Track the below URL for one-step submission https://juniperpublishers.com/online-submission.php 